\title{
Microwave Aquametry of Roasting Coffee Beans
}

\author{
Cameron Kelly, Jonathan Scott
}

\begin{abstract}
We constructed a fluidsed-bed coffee roaster with a roast chamber that is also a microwave cavity in order to measure water content of roasting coffee. We identify factors involved in roasting that influence the data. We present a new approach to the problem of interpreting measurements that are noisy as a result of bean movement in the fluidised bed. Cleaned data permit aquametry throughout the roast. We seek the ideal point when an expert roaster would terminate the roast, but this measurement found no events or conditions that signal the ideal endpoint for a roast.
\end{abstract} Bed

Index Terms-Coffee Roasting, Microwave Aquametry, Fluid

\section{INTRODUCTION}

C OFFEE roasting is an important industrial process where the coffee undergoes many chemical and physical changes not all of which are fully understood $[1,2]$. Over the last decades, machines for grinding and brewing moved from factories to the home, but roasting has not. It is our ultimate aim to automate the roasting process. The moisture content of beans is known to fall from $9-15 \%$ to about $1-2 \%$ during an expert roast [2]. In this work we investigate the possibility of achieving an appropriate roasting duration, finding the correct end-point, by measuring the moisture present in beans using microwaves during the coffee roast.

\section{A. Microwave Aquametry}

Microwave moisture measurement techniques fall into four categories [3, 4]: the 1-port open-ended method [5], the 2-port transmission method [6], and 1- or 2-port resonant methods [7]. All utilise the dielectric properties of water. We describe a method based on the 1-port resonant method.

\section{B. Notes on coffee roasting}

Coffee roasting is the process that takes green coffee beans and by the application of heat produces all the characteristic colours and flavours for which coffee is known. The initial phase of coffee roasting involves the heating and evaporation of moisture in the coffee. The next phase is roasting. During this stage the coffee undergoes over two hundred chemical reactions producing the changes in colour and flavour. This phase ends when the coffee starts to blacken and char; producing burnt tasting coffee. Each of these stages are punctuated with well known way-points called first and second crack.

First crack is where the coffee pops, in a manner similar to popcorn, due to a build up of moisture and other volatiles inside the bean. Second crack happens at the end of roasting when the reactions become so energetic that it causes the breaking down of the cell walls. Second crack can be so energetic that pieces of the bean are blown off. Coffee roasted past second crack is usually considered burnt. The art of coffee roasting is knowing when ending the roast will produce the desired flavors. A typical goal is to finish roasting before second crack occurs to maximise the roast flavours while avoiding a burnt flavour.

\section{Coffee RoAsting Design And EQuipment}

For this experiment we built a small scale fluidised-bed coffee roaster. A fluidised-bed roaster roasts coffee by blowing hot air though the coffee. The air flow causes the beans to move and flow as if they were in a fluid. We chose the fluid bed method of roasting because it allowed us to precisely control the air temperature surrounding the coffee.

Our coffee roaster was designed to roast a small batch of green coffee (roughly 66 grams) whilst being able to control the temperature of the air that the coffee is roasting in. The mechanical section of our roaster consists of a blower piped into a heating element. The hot air is then ducted in the base of a roasting chamber. Figure 1 displays a summary of the coffee roaster, control, and measure systems.

Our choice of measurement approach was limited because we were taking moisture measurements while simultaneously roasting coffee. We designed a roasting chamber in which both functions could be facilitated (Figure2). We chose a 1port resonator as our measurement method. This was mostly because of the physical constraints. Our roast chamber is made of stainless steel and acts as the resonant chamber. The hot air for roasting is pumped through perforated stainless steel disks at both the top and bottom of the chamber. The microwave energy is admitted in the side wall connected to a coupling structure. As our roast chamber is not ideal for making moisture measurements we did not seek to measure the absolute moisture or permittivity of the coffee. We wanted to identify the loss of moisture in the coffee by watching the change in frequency and impedance of a resonant peak during the roast.

Our goal is to be able to identify the shifting moisture levels in the coffee during roast. We identified the factors likely to have major influence on our measurements as: the change in moisture, change in temperature, and movement of the beans. We conducted preliminary tests in our chamber to observe the effects that these factors have on our measurements. We expect to see any changes in permittivity displayed as a shift in a resonant peak of the chamber.

\section{MEASUREMEnT FACTORS}

The clearest resonant peaks, most sensitive to the above factors, were visible in the real part of $\mathrm{Z}$ measurements at $3.5 \mathrm{GHz}$ and $4.0 \mathrm{GHz}$. The $3.5 \mathrm{GHz}$ peak was the largest but it was surrounded by other peaks which it would overlap with 


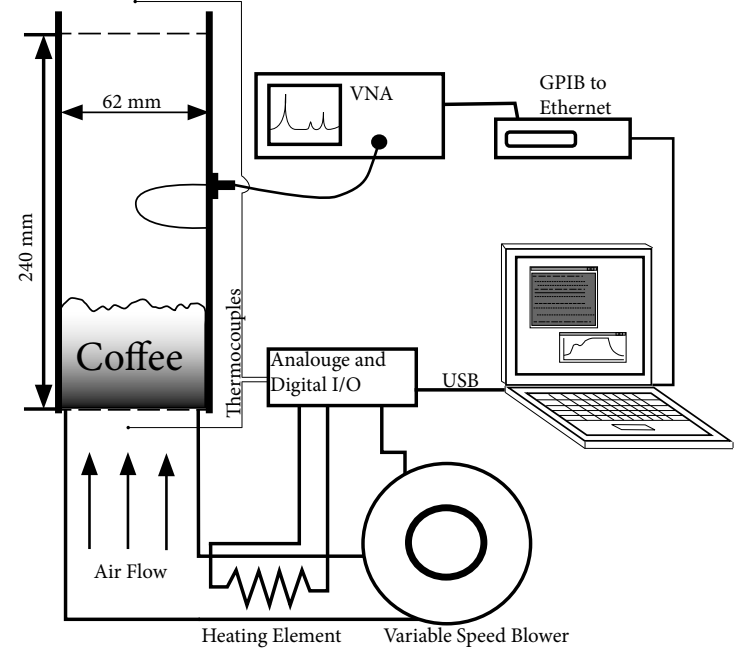

Fig. 1. Block diagram of the fluidised-bed roast chamber and attached equipment

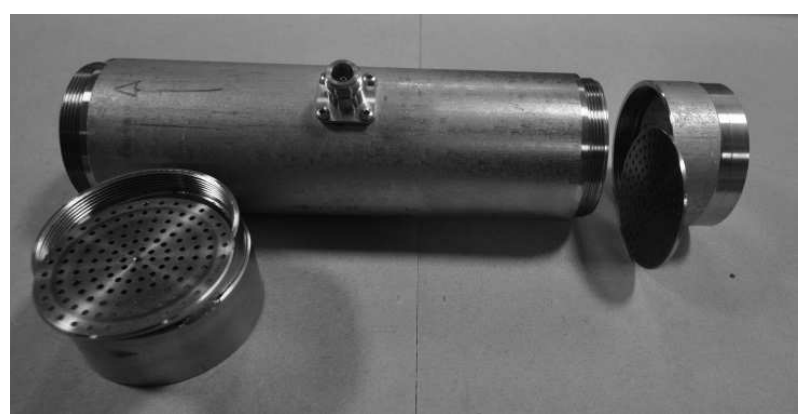

Fig. 2. Image of the stainless steel roasting chamber

during roasting. The $4 \mathrm{GHz}$ peak was conveniently isolated from other peaks so was used for our measurement.

The major factors that affect the permittivity measurements in our coffee roasting are bean movement, bean moisture content, and bean temperature. The movement of the beans introduced a large amount of "noise" to the system. Noise created by the bean movement only increased over the course of the roast as the decrease in bean density led to an increase in bean movement. We present a method of dealing with this noise in the result section.

The coffee used in our tests was a wet-processed Costa Rican Arabica. The coffee was measured-by drying-to have a moisture content of $11.5 \%$. We were able to observe the effect that temperature had on the resonant peaks by suspending varying amounts of water in a series of bean size sponges. We found that an increase in moisture content caused the resonant peak to attenuate and lower in frequency. We also found in comparing straight water in a sponge to the moisture contained in coffee beans that similar masses of water suspended in the different mediums affected the resonant peak to different levels, with the sponge water having a more pronounced effect. We attribute this to the reduced dipole moment of water trapped in the molecular structure of the coffee bean.

In our roasting tests the coffee was heated using a set temperature profile; the temperature of the input air is presented in Figure 3. We observed the effect of temperature on the resonant peak by observing measurements taken of coffee roasted to high temperature, $250 \mathrm{C}$, as it cooled to $40 \mathrm{C}$. We found that an increase in temperature both attenuates the resonant peak and lowers its frequency.

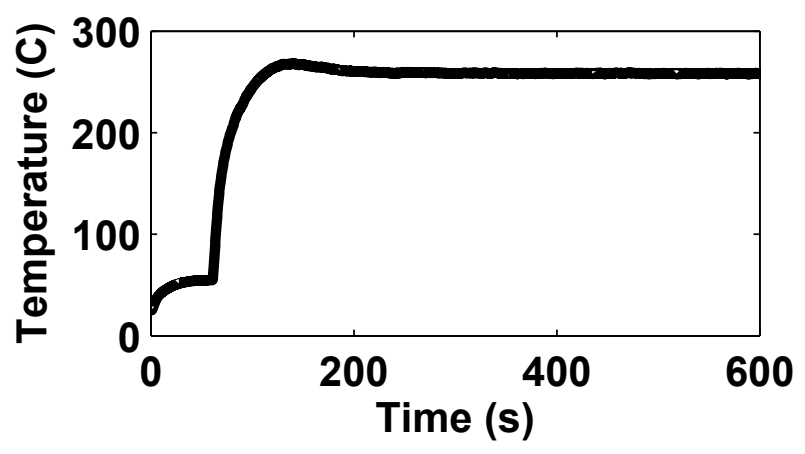

Fig. 3. Roaster roast air temperature profile

\section{Coffee RoAsting Results}

During a typical roast the coffee experienced first crack between 250 and 350 seconds. Second crack started around 550 seconds and was still continuing at the end of the roast. Figure 4 shows the measurement of the $4 \mathrm{GHz}$ peak during a typical roast. Initially the peak is very well defined. As the temperature increases the peak falls in frequency and amplitude. By 150 seconds the peak has attenuated to the point that it is barely distinguishable from the background. After this point the peak starts to return and shifts back toward its starting point. At around 250 seconds the peak starts to get very noisy and moves around, becoming harder to follow visually.

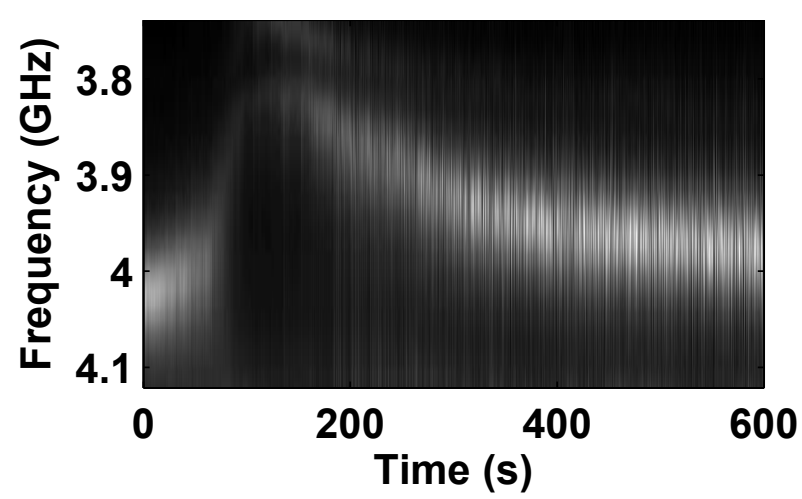

Fig. 4. This graphic show the $4 \mathrm{GHz}$ resonant peak during a typical roast. Each column represents a single network analyser trace. The gradient going from black approaching 0 and white being the peaks maximum.

When first crack occurs the beans undergo a rapid change in density. Accompanying this rapid change is an increase in the movement in the coffee. This change can be seen in our measurements when the signal becomes more noisy from the 250 second mark. Unfortunately for our goal-making 
predictions of the ideal end of roast - the point in which we are interested occurs after first crack. We therefore needed a method of making sense of the data in the noisy post-first crack region.

We developed a technique to make sense of the data by tracking the frequency of the resonant peak over the roast. The technique worked by isolating the peak in which we are interested. The process started with a five point running average against time. This smoothed the data and helped remove any transient outliers. We also ran a low-pass filter on the frequency component of each trace. The third step was to run a peak detection algorithm against the adjusted and filtered data.

We added an extra step to further isolate the resonant peak's path during the roast (Figure 5). This technique allowed us to identify the resonant peak and track it through the roast. The cleaned data is more useful in making observations of what is happening during roasting.

What we observe is the change in temperature and the change in moisture working against each other. The large initial temperature increase causes a rise in permittivity, lowering the resonant peak's frequency. As the coffee heats up it starts to lose moisture causing a loss in permittivity. As the temperature increases the rate of moisture loss also increases. The rate of moisture loss eventually overcomes the increase in temperature and the peak starts shifting back. For the second half of the roast (the region we are interested in) the temperature stabilises and we observe the peak increasing in frequency. This increase we can attribute to the coffee beans' continued loss in moisture.

These measurements are consistent with what we expect from roasting coffee. Unfortunately they do not show any change of trend, definitive sign, or transient that could be used as an indicator for the ideal end of roast.

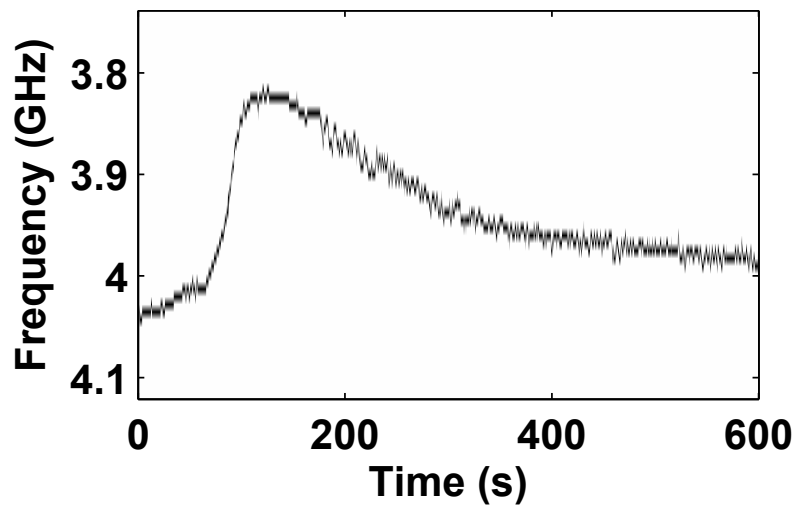

Fig. 5. This figure shows the result of the peak isolation method applied to the $4 \mathrm{GHz}$ peak. It displays the peak's movement in frequency over the course of the roast.

\section{CONCLUSION}

We proposed a method of measuring and controlling coffee roasting using microwave aquametry. We built a microwave moisture meter to make direct measurements of coffee while roasting in a fluidised bad. The technique was successful in observing the change in moisture content. We were able to identify the effects of both temperature and moisture in preliminarily tests and then observe the affects of these two factors on the roasting coffee. Although states in the roast could be identified using the measurements we could not identify the end roast using this method.

\section{REFERENCES}

[1] A. Illy and R. Viani. Espresso Coffee, Second Edition: The Science of Quality. Academic Press, Jan. 2005.

[2] R. J. Clarke and R. Macrae. Coffee, Volume 2: Technology. 1st ed. Elsevier Applied Science, Feb. 1987.

[3] A.W. Kraszewski. "Microwave aquametry-needs and perspectives". In: IEEE Transactions on Microwave Theory and Techniques 39.5 (1991), pp. 828-835.

[4] A.W. Kraszewski and S.O. Nelson. "Microwave permittivity determination in agricultural products". In: 12th International Conference on Microwaves and Radar, 1998. MIKON '98. Vol. 4. 1998, 230-242 vol.4.

[5] B. Filali et al. "Design and Calibration of a Large Open-Ended Coaxial Probe for the Measurement of the Dielectric Properties of Concrete". In: IEEE Transactions on Microwave Theory and Techniques 56.10 (2008), pp. 2322-2328.

[6] A.M. Nicolson and G. F. Ross. "Measurement of the Intrinsic Properties of Materials by Time-Domain Techniques". In: IEEE Transactions on Instrumentation and Measurement 19.4 (1970), pp. 377-382.

[7] A.W. Kraszewski, S.O. Nelson, and T.-S. You. "Use of a microwave cavity for sensing dielectric properties of arbitrarily shaped biological objects". In: IEEE Transactions on Microwave Theory and Techniques 38.7 (1990), pp. $858-863$. 